\title{
CRANIOCEREBRAL INJURIES FROM DOG BITES
}

\author{
Fernando Gomes Campos Pinto², Wagner Malago Tavares', Daniel Dante Cardeal', \\ Edson Bor-Seng-Shu', Almir Ferreira de Andrade ${ }^{2}$, Manoel Jacobsen Teixeira ${ }^{2}$
}

Dog bites are increasingly being recognized as posing a major public health problem. In the U.S., Weiss et al. reported a rate of 12.9 per 10,000 persons, for dog-bite related injuries resulting in a total of 333,687 visits to Emergency Departments'. Children most commonly fall victim, where the head and neck are the most frequent sites of injury accounting for some $80 \%$ of such cases.

To date, craniocerebral injury cases caused by dog bites have been little published in the medical literature ${ }^{2,3}$. Two cases of penetrating head injury from dog bites are presented and discussed in the context of the current literature on clinical and radiological evaluation, and treatment.

\section{CASE}

This study was approved by the commission of ethics of the institution and we obtained the informed consent of the responsible for the patients for publication.

\section{Case 1}

On January, 2006 a previously healthy two-year-old boy was brought to the "Hospital das Clínicas" of São Paulo 12 hours after being attacked by a Rotweiller dog in the head. The boy did not lose consciousness, remained alert and responded appropriately. Examination showed a scalp injury on both sides of the head. The CT scan disclosed a depressed skull fracture in the right temporal bone, where the scalp injury was larger (Fig 1A). The child was submitted to surgery with a large incision. There were five depressed skull fractures in the temporal and parietal bones, not directly under the scalp injury itself, which were repaired with a wide craniotomy. The next day the child presented CSF leakage on the left side from the scalp injury, and underwent surgery for a second time. The second CT scan showed a skull fracture in the left temporal bone, not revealed in the first CT scan (Fig 1B). Surgery was carried out on this side and two depressed skull fractures were found not under the scalp wound, but some distance away (Fig 1C, 1D). A large craniotomy was performed and the dural laceration was treated. The boy received a wide spectrum prophylactic antibiotic (oxacilin, ceftriaxone and metronidazol) for 14 days, anti-rabies and anti-tetanus vaccines, rabies immunoglobulin, where treatment was complemented

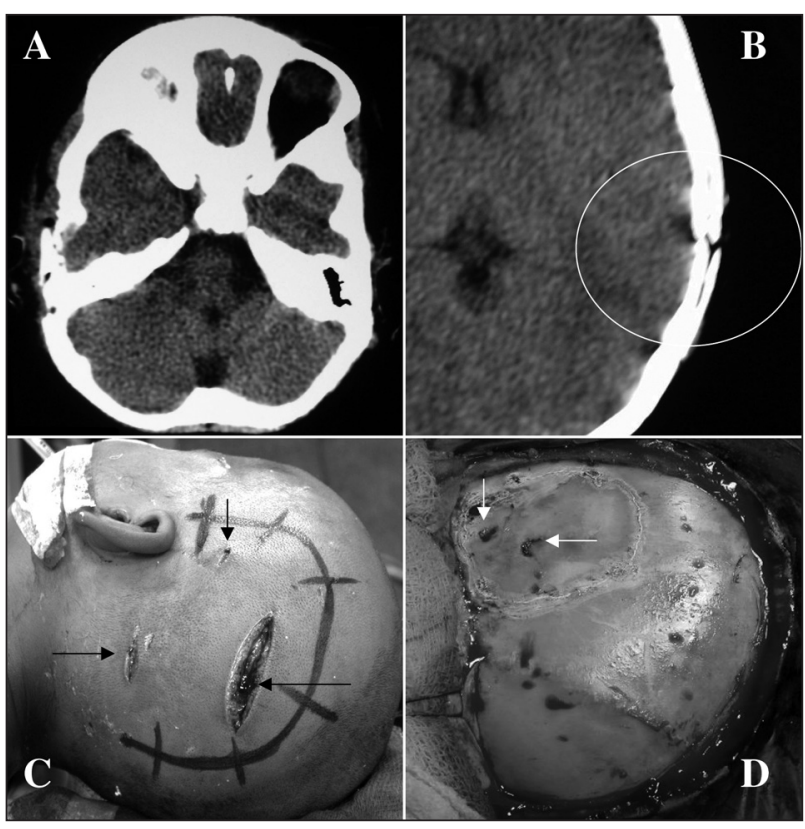

Fig 1. Case 1. (A) First CT scan - a depressed skull fracture in the right temporal bone; (B) Second CT scan - a skull fracture in the left temporal bone; (C) Second surgery - 3 injuries to scalp $(\rightarrow)$; (D) two depressed skull fractures in the left temporal bone $(\rightarrow)$.

with psychological therapy. At a twelve month-follow-up, the boy was normal without any handicap.

\section{Case 2}

On May, 2006 a previously healthy three-year-old girl was brought to the "Hospital das Clínicas" of São Paulo 10 hours after she had been attacked to the head by a pitbull dog. She did not lose consciousness and presented scalp injuries on both sides of the head as well as to the posterior region of the head. The girl was conscious and responded appropriately. The CT scan showed a complex depressed skull fracture in the left temporal bone; two depressed skull fractures in the occipital bone and signs of pneumocephalus. The 3D CT showed both bone and brain injuries (Fig 2). The girl was submitted to surgery with wide exposure of the lesions. Fractures and dural lacerations were corrected and (Figs 3 and 4). After surgical treatment she received prophylactic antibiotics (oxacilin, ceftriaxone and metronida-

\section{TRAUMATISMO CRANIENCEFÁLICO POR MORDIDA DE CACHORRO}

Division of Neurosurgery, University of São Paulo, São Paulo SP, Brazil: ${ }^{1} \mathrm{MD} ;{ }^{2} \mathrm{MD}$, PhD.

Received 8 February 2008, received in final form 22 April 2008. Accepted 5 May 2008.

Dr. Fernando Campos Gomes Pinto - Rua Bartira 1060 / 61 - 05009-000 São Paulo SP - Brasil. E-mail: fernando.neuro@terra.com.br 

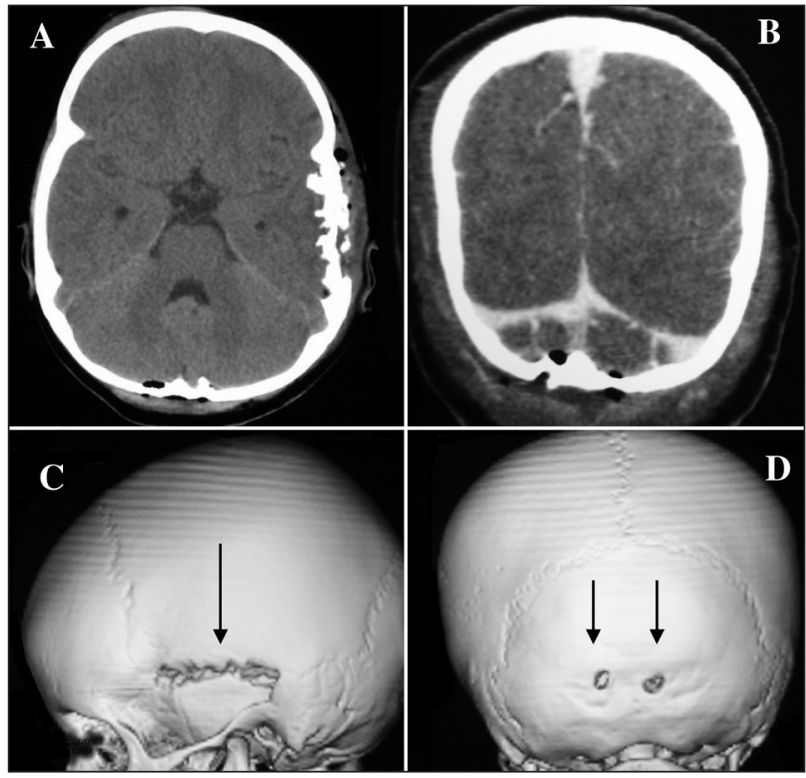

Fig 2. Case 2. (A) CT scan - a complex depressed skull fracture in the left temporal bone and two depressed skull fractures in the occipital bone; (B) Coronal CT with contrast - two depressed skull fractures in the occipital bone below the transverse sinus; (C) $3 D C T$ - a complex depressed skull fracture in the left temporal bone $(\rightarrow)$; $(D) 3 D C T$ two depressed skull fractures in the occipital bone $(\rightarrow)$.

zol) for 14 days, anti-rabies and anti-tetanus vaccine, rabies immunoglobulin and the treatment was complemented with psychological therapy. After a follow-up of twelve months, the girl was normal without any handicap.

\section{DISCUSSION}

The most common sites of injury caused by dog bites in children are the face, head and neck, which account for $73 \%$ of cases, against $30 \%$ in adults in the same regions ${ }^{4-6}$. Mitchell et al. showed that the attack site was familiar to the child in $59 \%$ of cases ${ }^{2}$. Although CDC Injury Center found 25 breeds to be involved in attacks, the pit bull and rottweiler breeds were responsible for $50 \%$ of all cases.
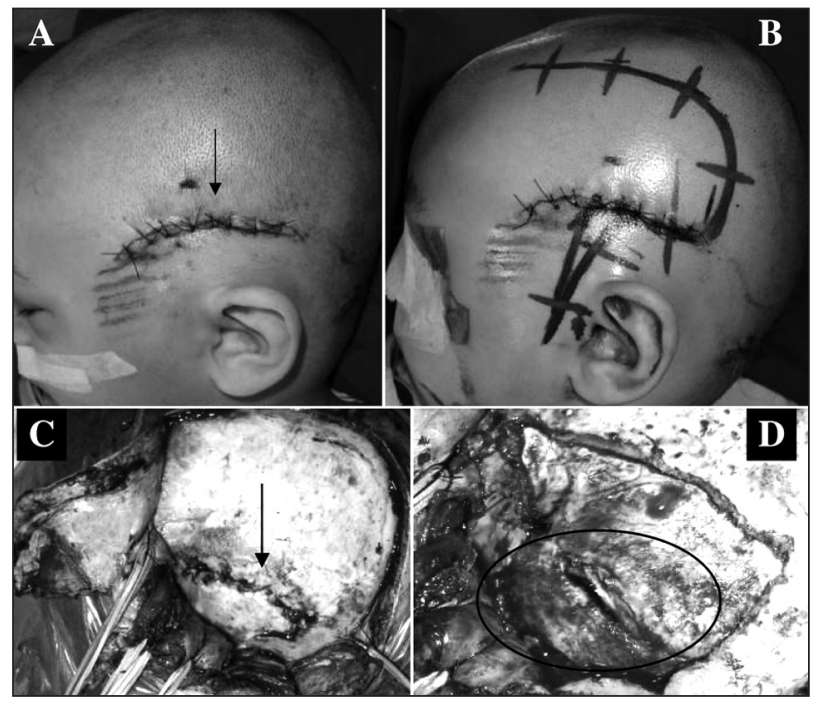

Fig 3. Case 2. (A) Scalp injury to left temporal area ( $\rightarrow$ ); (B) Surgical planning; (C) A complex depressed skull fracture in the left temporal bone $(\rightarrow)$; (D) Temporal dural laceration with CSF fistula (circle).

Their jaws can generate pressures of about 200 to 400 sq. lb/psi., sufficient to perforate sheet metal ${ }^{7}$.

There are several reasons why young children are more likely than adults to be attacked to the head. First, children's lower stature. Second, children are more likely to lean/hold their faces close to the dog's head which is perceived as a threatening distance by dogs. Third, adults tend to provide a better defense against attacks.

The few symptoms and apparent clinical and neurological stability of present cases may give a false sense of lower severity. However, the patients presented small skull fractures, largely punctiforms due to canine teeth penetration, associated with little apparent skin and duramater lesions. Additionally, routine head CT scan were not able to disclose the small fractures exacerbating this false sense of security.


Fig 4. Case 2. (A) Scalp injuries to occipital area ( $\rightarrow$ ); (B) Surgical planning; (C) Two depressed skull fractures in the occipital bone; (D) Two dural lacerations with CSF fistula $(\rightarrow)$. 
In the first case the child was submitted to surgery and the site of brain injury and CSF leak was identified. However, after 24h, we found CSF on the contralateral site that had not previously been recognized as there was no evidence of CSF fistula on admission, while the conventional CT scan had not shown any signs of fracture. Despite appearing only a puncture lesion, wide opening and craniotomy must explore pediatric patients with scalp injury from dog bites. The site of scalp injury often differs to the site of the skull fracture, probably by reason of the soft subcutaneous tissue and the distraction caused by the dog jaw. Thorough scalp debridment and extensive irrigation must be conducted.

In the second case we performed a head CT scan with $3 \mathrm{D}$ reconstruction that promptly showed the sites of the fractures which again could not be seen on the axial CT scan. Because of its higher resolution and sensitivity for this kind of fracture we prefer 3D CT scan over conventional X-rays.

Current recommendations for antibiotic prophylaxis are controversial. Smith et al. showed that there is poor evidence of benefit, while other authors have suggested using antibiotics in any dog bite injury with bone involvement, especially if there is CSF inoculation $2,3,8,9$. If infection does occur, Pasteurella multocida is isolated in culture in more than $50 \%$ of the cases, followed by Staphylococcus $(25 \%)$ and Streptococcus $(15 \%)^{8}$. Our cases both received antibiotic therapeutic regimens with oxacillin, ceftazidime and metronidazol for 14 days. All patients must be checked for tetanus, and be submitted to immunoglobulin treatment if necessary. Anti-rabies vaccination and rabies immunoglobulin are mandatory given the nature of the injury.

Almost half of all children suffering a dog attack develop posttraumatic stress disorder ${ }^{10}$. Both of our child cases were followed up with psychological support.

\section{REFERENCES}

1. Weiss HB, Friedman DI, Coben JH. Incidence of dog bite injuries treated in emergency departments. JAMA 1998;279:51-53.

2. Mitchell RB, Nanez G, Wagner JD, Kelly, J. Dog bites of the scalp, face, and neck in children. Laryngoscope 2003;113:492-495.

3. Peters V, Sottiaux M, Appelboom J, Kahn A. Posttraumatic stress disorder after dog bites in children. J Pediatr 2004;144:121-122.

4. Iannelli A, Lupi G. Penetrating brain injuries from a dog bite in an infant. Pediatr Neurosurg 2005;41:41-45.

5. Tu AT, Girotto JA, Singh N, et al. Facial fractures from dog bite injuries. Plast Reconstr Surg 2002;109:1259-1265.

6. Wilberger JE Jr;Pang D. Craniocerebral injuries from dog bites. JAMA 1983;249:2685-2688.

7. Koplan JP. The Injury Fact Book 2001-2002. Atlanta, GA: National Center for Injury Prevention and Control, 2002:61-63.

8. Callaham M. Dog bite wounds. JAMA 1980;244:2327-2328.

9. Davis SJM, Valla FR. Evidence for domestication of the $\operatorname{dog} 12,000$ years ago in the Natufina of Israel. Nature 1978;276:608-610.

10. Smith MR, Walker A, Brenchley J. Barking up the wrong tree? A survey of dog bite wound management. Emerg Med J 2003;20:253-255. 